\title{
RESEARCH
}

\section{A survey of radiographic prescription in dental implant assessment}

\author{
CE Sakakura ${ }^{1}$, JAND Morais ${ }^{1}$, LCM Loffredo $^{2}$ and G Scaf*,1 \\ ${ }^{1}$ Department of Diagnosis and Surgery, Araraquara Dental School, State of São Paulo University, Unesp, Araraquara, São Paulo, \\ Brazil; ${ }^{2}$ Department of Social Dentistry, Araraquara Dental School, State of São Paulo University, Unesp, Araraquara, São Paulo, \\ Brazil
}

\begin{abstract}
Objectives: To survey the current radiographic prescriptions in dental implant assessment amongst dentists in Brazil.

Methods: Sixty-nine dentists were interviewed during a dental implant meeting by two calibrated graduate students, using a 19-question questionnaire, considering imaging modality options both for pre-operative implant site assessment and for follow-up, particularly with respect to cost, patient radiation dose, and broad coverage of facial bones and teeth. Epi-Info 6.04 software was used to analyse the database file.

Results: Approximately $63.8 \%$ of the dentists prescribed only panoramic radiography for dental implant assessment and $28.9 \%$ ordered panoramic radiography plus periapical radiography and/or conventional tomography and/or computed tomography (CT). Only $7.2 \%$ of the dentists ordered conventional tomography or CT as a single examination, although $10.1 \%$ ordered it in combination with other imaging modalities. The main reasons given for prescribing panoramic radiography were broad coverage and cost $(86.4 \%)$.

Conclusions: This study has shown that most of the dentists in this study prescribe panoramic radiographs in dental implant assessment based on broad coverage and cost. They are not following the American Academy of Oral and Maxillofacial Radiology recommendations regarding cross-sectional imaging.

Dentomaxillofacial Radiology (2003) 32, 397-400. doi: 10.1259/dmfr/20681066
\end{abstract}

Keywords: survey; dental radiography; dental implants; tomography, X-ray computed

\section{Introduction}

The use of osseointegrated implants is a widely accepted procedure in the rehabilitation of edentulous spaces. ${ }^{1,2}$ Treatment planning for implants includes a radiographic examination that provides information about the location of anatomical structures, the quality and quantity of available bone, the presence of bone lesions, ${ }^{1-4}$ the occlusal pattern, and the number and size of implants, as well as prosthesis design, all of which are essential for successful implant treatment.,3 Many types of radiographic imaging are recommended for treatment planning for implants, such as panoramic, periapical and occlusal radiographs, conventional tomography and computed

*Correspondence to: Gulnara Scaf, Departamento de Diagnóstico e Cirurgia, Faculdade de Odontologia de Araraquara, Unesp, Rua Humaitá, 1680, CP: 331, 14.801-903 - Araraquara, São Paulo, Brazil; E-mail: scaf@ foar.unesp.br Received 24 April 2003; revised 1 October 2003; accepted 10 October 2003 tomography (CT). The clinicians need to identify the best method for each clinical situation. ${ }^{4-9}$

Panoramic radiography is readily available and provides a view of many structures of the maxilla and mandible at a low cost. However, image magnification and lack of cross-sectional information are the major disadvantages of this image modality for treatment planning. In spite of these disadvantages, panoramic radiography has been the most common single radiographic examination used in implant treatment planning.

Beason and Brooks ${ }^{10}$ determined the types of imaging used for pre-operative implant site assessment in a random sample of dentists in south-east Michigan, USA. The results showed that more than $95 \%$ of the dentists take panoramic radiographs on at least $80 \%$ of patients. Over 90\% responded that they never prescribe conventional tomography, although the American Academy of Oral and 
Maxillofacial Radiology (AAOMR) ${ }^{9}$ recommends crosssectional imaging for implant site evaluation by either conventional tomography or CT.

Since there is little information about how dentists in other countries prescribe radiographs for dental implant assessment, the aim of this study was to survey the current radiographic prescription for this task in Brazil and to compare it with the AAOMR recommendations.

\section{Material and methods}

Two graduate students (CES and JANDM), previously trained and calibrated in the use of the questionnaire, interviewed a random sample of 69 dentists attending a dental implant meeting held in São Paulo, Brazil. All of the selected dentists undertook surgical planning of dental implant treatment. They included the following specialties: Implantology (50.8\%), Periodontology (27.9\%), General Dentistry (14.8\%), Oral Surgery (4.9\%) and Prosthodontics $(1.6 \%)$. A closed-end questionnaire with single answers to 19 questions was prepared to collect data about the types of radiographic examinations the dentists prescribe for pre-operative implant site assessments and follow-up, such as panoramic radiography, CT, conventional tomography and periapical radiography, either alone or in combination. They were also asked the reasons for their choice, including cost, patient radiation dose, broad coverage of facial bones and teeth, availability and measurement precision. There was no specific question regarding need or desire for cross-sectional imaging. Before the study began, a pre-test of the questionnaire was performed with the participants of an oral implantology continuing education course at the Araraquara Dental School, Unesp.

The database file of survey answers was analysed by Epi-Info 6.04 software.

\section{Results}

The results are shown in Figures 1 and 2 and in Table 1.

The radiographic examinations more often prescribed for dental implant assessment were the panoramic radiograph $(63.8 \%)$, followed by panoramic plus periapical radiographs (18.8\%). Approximately $7.2 \%$ of the dentists prescribed CT or conventional tomography as a single examination, and $10.1 \%$ preferred the combination of CT or conventional tomography with other types of radiographic methods (Figure 1 ).

The most common reason for prescribing a specific radiographic examination was the desire for broad coverage of the facial bones and teeth $(37.7 \%)$, followed by measurement precision $(14.5 \%)$, cost $(13.0 \%)$ and availability $(5.8 \%)$. More than half of the reasons $(60.8 \%)$ were broad coverage, cost or the combination of both. The least common reason given was the low radiation dose of the technique (1.4\%) (Figure 2).

Table 1 shows the reasons given for prescribing each specific examination or combination. The main reasons to prescribe a panoramic radiograph were broad coverage $(52.3 \%)$ and cost $(18.2 \%)$. The combination of both these reasons was $15.9 \%$. The main reason to prescribe conventional tomography or CT, alone or in combination with panoramic and periapical radiography, was the precision of the measurements (Table 1).

\section{Discussion}

The decision to survey the current radiographic prescription in dental implant assessment was in order to determine whether dentists are using cross-sectional imaging for treatment planning for implant placement as recommended by the AAOMR. ${ }^{9}$

In this study, the panoramic radiograph was the most frequent radiographic examination prescribed for treatment planning of osseointegrated implants. Approximately, $82.6 \%$ of dentists prescribed the panoramic radiograph, either as a single examination or combined with a periapical radiograph. These results show that the dentists have not been using cross-sectional imaging, taking the risk of damaging anatomical structures, mainly the inferior alveolar nerve. ${ }^{5,6}$ Beason and Brooks ${ }^{10}$ found similar results: over $95 \%$ of the dentists in their survey took panoramic radiographs on at least $80 \%$ of their patients.

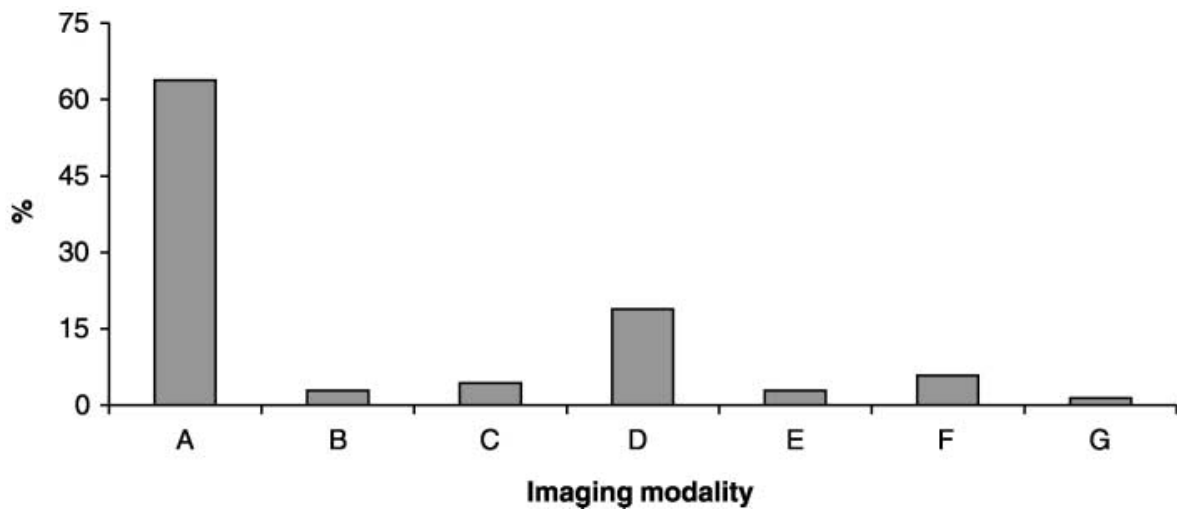

Figure 1 Distribution of the imaging modality options in dental implant assessment. A, panoramic radiography; B, computed tomography (CT); $\mathrm{C}$, conventional tomography; $\mathrm{D}$, panoramic + periapical radiography; E, panoramic radiography $+\mathrm{CT}$; $\mathrm{F}$, panoramic radiography + conventional tomography; G, panoramic + periapical radiography + conventional tomography 


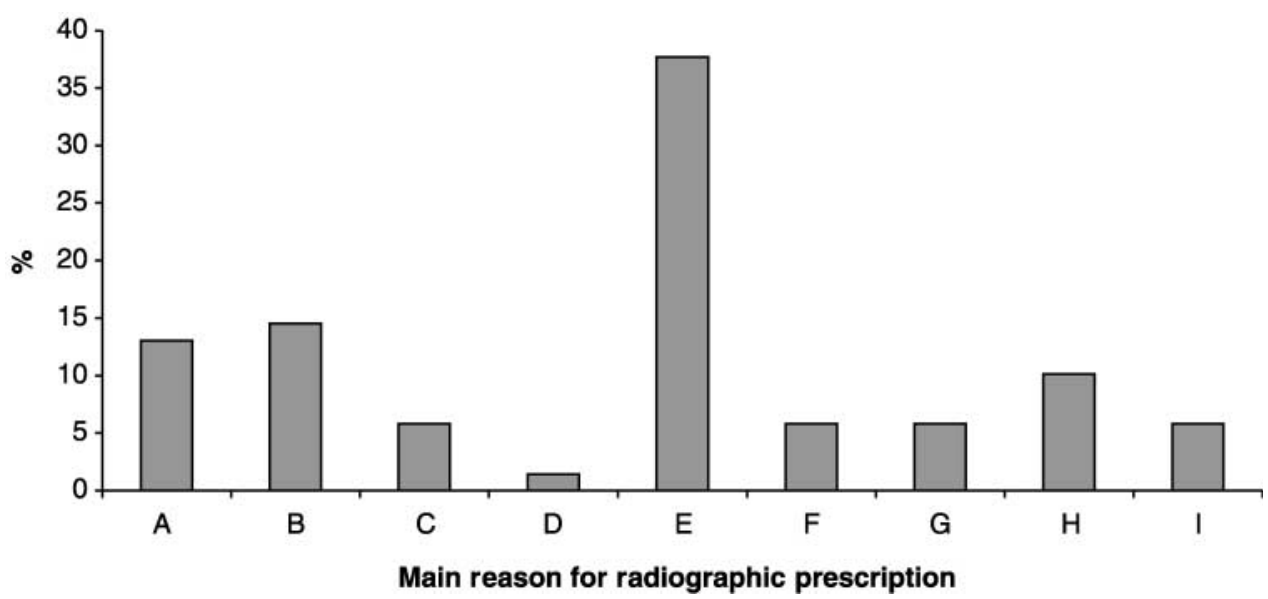

Figure 2 Distribution of the main reasons for prescribing radiographs for dental implant assessment. A, cost; B, measurement precision; C, availability; D, low radiation dose; E, broad coverage; F, cost + measurement precision; G, cost + availability; H, cost + broad coverage; I, other reasons

Table 1 Relationship between the type of radiographic examination and the reasons for prescribing it for dental implant assessment

\begin{tabular}{|c|c|c|c|c|c|c|c|c|c|c|}
\hline \multirow[b]{2}{*}{ Radiographic examination } & \multicolumn{9}{|c|}{ Reasons } & \multirow[b]{2}{*}{ Total } \\
\hline & $A$ & $B$ & $C$ & $D$ & $E$ & $F$ & $G$ & $H$ & $I$ & \\
\hline Panoramic radiography & 8 & 0 & 1 & 1 & 23 & 1 & 3 & 7 & 0 & 44 \\
\hline CT & 0 & 2 & 0 & 0 & 0 & 0 & 0 & 0 & 0 & 2 \\
\hline Conv. tomography & 0 & 2 & 0 & 0 & 0 & 1 & 0 & 0 & 0 & 3 \\
\hline Panoramic radiography + periapical radiography & 1 & 1 & 3 & 0 & 3 & 2 & 0 & 0 & 3 & 13 \\
\hline Panoramic radiography $+\mathrm{CT}$ & 0 & 2 & 0 & 0 & 0 & 0 & 0 & 0 & 0 & 2 \\
\hline Panoramic radiography + conv. tomography & 0 & 2 & 0 & 0 & 0 & 0 & 1 & 0 & 1 & 4 \\
\hline Panoramic radiography + periapical radiography + conv. tomography & 0 & 1 & 0 & 0 & 0 & 0 & 0 & 0 & 0 & 1 \\
\hline Total & 9 & 10 & 4 & 1 & 26 & 4 & 4 & 7 & 4 & 69 \\
\hline
\end{tabular}

A, cost; B, measurement precision; C, availability; D, low radiation dose; E, broad coverage; F, cost + measurement precision; G, cost + availability; $\mathrm{H}$, cost + broad coverage; I, other reasons

CT, computed tomography; Conv., conventional

The panoramic radiograph gives useful information in the initial evaluation for pre-operative planning, but there is a large horizontal magnification in this type of image, which varies depending on the region. For this reason, measurements are not recommended on panoramic radiographs. Another limitation of panoramic radiography is the lack of information in the third dimension. $5,6,9,11$

More than half of the dentists prescribed a panoramic radiograph because of broad coverage (52.3\%), followed by cost $(18.2 \%)$, and the combination of coverage and cost (15.9\%). Only one dentist $(2.3 \%)$ who prescribed panoramic radiography indicated that it was because of precision of the measurements, which is crucial for pre-operative surgical planning. Although we did not specifically ask about the need or desire for cross-sectional information, we assume that dentists are not worried about this since so few selected either conventional tomography or CT. Based on our implant surgery experience, we speculate that dentists are self-confident about their performance regarding clinical and surgical procedures as a result of their professional experience in detecting bone thickness and performing the surgical procedures with a safety margin and thus avoiding surgical damage. However, lack of cross-sectional information can lead to the use of shorter and narrower implant sizes, ${ }^{12}$ which may impair the stress distribution.

In this study, only $7.2 \%$ of the dentists prescribed conventional tomography or $\mathrm{CT}$ as a single examination, and $10.1 \%$ prescribed CT in combination with other types of radiographic methods, giving a total of $17.3 \%$ using some type of cross-sectional imaging. Our results are similar to Beason and Brooks. ${ }^{10}$ They found that more than $90 \%$ of dentists had never used conventional tomography and $65 \%$ had never used CT. These results show that a minority of dentists use cross-sectional imaging for implant placement.

The main reasons for not prescribing cross-sectional imaging are probably related to the high cost and limited facilities in small towns compared with panoramic radiography, although the panoramic machine is not available in all private dental practices. If crosssectional information is desired, it can be obtained at lower cost with conventional tomography than with $\mathrm{CT}^{7}$

Another aspect to be considered in the implant imaging prescription is the radiation dose. ${ }^{7,11}$ In this study only one dentist $(1.4 \%)$ indicated that radiation dose influenced the choice of imaging examination. We were surprised by this result, because dose reduction in stressed in dental schools. 
Although panoramic radiography requires only a small radiation dose, it does not provide information in the third dimension, which is deemed necessary by some. Following the ALARA (as low as reasonable achievable) principle, for cross-sectional imaging the $\mathrm{AAOMR}^{9}$ recommends conventional tomography for one to seven implant sites and CT for eight or more implant sites.

Besides the reasons stated above regarding the low utilization of cross-sectional imaging by clinicians, we speculate that one of the reasons why more dentists do not use conventional tomography or CT is the difficulty of image interpretation. Attempts must be made to solve these problems, changing the educational programmes in continuing education courses.

\section{References}

1. Adell R, Lekholm U, Rockler B, Branemark PI. A 15-year study of osseointegrated implants in the treatment of the edentulous jaw. Int $J$ Oral Surg 1981; 10: 387-416.

2. Andersson B, Odman P, Lindvall AM, Branemark PI. Five-year prospective study of prosthodontic and surgical single-tooth implant treatment in general practices and at a specialist clinic. Int $J$ Prosthodont 1998; 11: 351-355.

3. Engelman MJ, Sorensen JA, Moy P. Optimum placement of osseointegrated implants. J Prosthet Dent 1988; 59: 467-473.

4. Stella JP, Tharanon W. A precise radiographic method to determine the location of the inferior alveolar canal in the posterior edentulous mandible: implications for dental implants. Part I: technique. Int J Oral Maxillofac Implants 1990; 5: 15-22.

5. Klinge B, Petersson A, Malay P. Location of the mandibular canal: comparison of macroscopic findings, conventional radiography and computed tomography. Int J Oral Maxillofac Implants 1989; 4: $327-332$.

6. Lindh C, Petersson A. Radiologic examination for location of the mandibular canal: a comparison between panoramic radiography and conventional tomography. Int J Oral Maxillofac Implants 1989; 4: $249-253$.
In conclusion, this study has shown that the majority of dentists sampled in Brazil prescribe panoramic radiographs for dental implant assessment based on broad coverage and cost, and that they are not following the AAOMR recommendations regarding cross-sectional imaging.

\section{Acknowledgment}

We would like to thank to CAPES (Coordenação de Aperfeiçoamento de Pessoal de Nível Superior) for the fellowship used to support this study.

7. Scaf G, Lurie AG, Mosier KM, Kantor ML, Ramsby GR, Freedman ML. Dosimetry and cost of imaging osseointegrated implants with film-based and computed tomography. Oral Surg Oral Med Oral Pathol Oral Radiol Endod 1997; 83: 41-48.

8. ten Bruggenkate CM, van der Linden LW, Oosterbeek HS. Parallelism of implants visualized on the orthopantomogram. Int J Oral Maxillofac Surg 1989; 18: 213-215.

9. Tyndall DA, Brooks SL. Selection criteria for dental implant site imaging: a position paper of the American Academy of Oral and Maxillofacial Radiology. Oral Surg Oral Med Oral Pathol Oral Radiol Endod 2000; 89: 630-637.

10. Beason RC, Brooks SL. Preoperative implant site assessment in southeast Michigan. J Dent Res 2001; 80: 137 (Abstr. 810).

11. Kassebaum DK, Nummikoski PV, Triplett RG, Langlais RP. Crosssectional radiography for implant site assessment. Oral Surg Oral Med Oral Pathol 1990; 70: 674-678.

12. Schropp L, Wenzel A, Kostopoulos L. Impact of conventional tomography on prediction of the appropriate implant size. Oral Surg Oral Med Oral Pathol Oral Radiol Endod 2001; 92: 458-463. 